\title{
Uncertainty Analysis of Concrete Structures Using Modified Latin Hypercube Sampling Method
}

\author{
In-Hwan Yang ${ }^{1)}$
}

(Received February 25, 2006, and Accepted June 30, 2006)

\begin{abstract}
This paper proposes a modified method of Latin Hypercube sampling to reduce the variance of statistical parameters in uncertainty analysis of concrete structures. The proposed method is a modification of Latin Hypercube sampling method. This analysis method uses specifically modified tables of random permutations of ranked numbers. In addition, the Spearman coefficient is used to make modified tables. Numerical analysis is carried out to predict the uncertainty of axial shortening in prestressed concrete bridge. Statistical parameters obtained from modified Latin Hypercube sampling method and conventional Latin Hypercube sampling method are compared and evaluated by a numeric analysis. The results show that the proposed method results in a decrease in the variance of statistical parameters. This indicates the method is efficient and effective in the uncertainty analysis of complex structural system such as prestressed concrete bridges.
\end{abstract}

Keywords: uncertainty, Latin Hypercube sampling, probabilistic analysis, simulation, creep.

\section{Introduction}

The terminology of uncertainty analysis or probabilistic analysis is widely understood to mean the study of probabilistic or statistical properties of the model output with respect to the input. ${ }^{1,2}$ To perform a probabilistic analysis with a numerical model, it is necessary to obtain model output for various values of the input variables. Several approaches to the probabilistic analysis have been developed as numeric analysis tools. ${ }^{3}$ Also, a review of different methods for uncertainty analysis has been provided by Novak et al. ${ }^{4}$ Monte Carlo simulation method is a conventional approach to probabilistic analysis. However, in practice, Monte Carlo simulations may be limited by constraints, computer capability and significant expense of computer runs in such complex structural system as prestressed concrete bridges. A prestressed concrete bridge is of a complex structural system because it is constructed in stepwise and its structural behavior is time-dependent due to the creep and shrinkage of concrete. An alternative approach is to use a constrained sampling technique. One such a scheme, which was developed by Iman and coworkers, ${ }^{5-7}$ is Latin Hypercube sampling method. It provides estimates of several statistical parameters such as mean value, standard deviation, and coefficient of variation. In addition, Novak, et al. ${ }^{8}$ and Olsson et al. ${ }^{9}$ have shown the effectiveness of LHS method in probabilistic problems.

The purpose of this study is to compare the estimates of statistical parameters obtained from a different number of

\footnotetext{
1) KCI member, Technical Research Institute, Daelim Industrial Co. Ltd.,

Seoul 110-732, Korea. E-mail:ypower@dic.co.kr

Copyright (C) 2006, Korea Concrete Institute. All rights reserved, including the making of copies without the written permission from the copyright proprietors.
}

simulations by using modified Latin Hypercube sampling method, and conventional Latin Hypercube sampling method. In this study, a modified method of Latin Hypercube sampling is proposed by which the variance of statistical parameters of outputs can be reduced more. The paper presents a basic theoretical reasoning sampling scheme. This method uses specially modified tables of random permutations of rank numbers, which form the input samples for a simulation procedure. Finally, numerical analyses are carried out to show that the proposed modification of Latin Hypercube sampling method can result in a significant decrease in the variance of the estimates of commonly used statistical parameters.

\section{Latin Hypercube sampling method}

Simulation is the process of replicating the real world based on a set of assumptions and conceived models of reality. It may be performed theoretically or experimentally. For engineering application, simulation may be applied to predict or study the performance and/or response of a system of interest. With a prescribed set of values for the system parameters (or design variables), the simulation process yields a specific measure of performance or response.

A conventional approach to this process is Monte Carlo simulation technique. However, in practice, Monte Carlo simulations may be limited by the significant cost of computer runs in complex structural system problems. An alternative approach is to use a constrained sampling scheme such as LHS method. ${ }^{5-7}$

LHS method consists of two steps to obtain a $N \times K$ design matrix. The first step is dividing each input variable into $N$ intervals. The second step is the coupling of input variables with tables of random permutation of rank numbers. Every input variable $X_{k},(k=1,2, \cdots, K)$ is described by its known cumulative 
function (CDF) $F_{X_{k}}(x)$ with the appropriate statistical parameters. The range of known CDF $F_{X_{k}}(x)$ of each input variable $X_{k}$ is partitioned into $N$ intervals with equal probability of $1 / N$.

The representative value in each interval is used just once during the simulation procedure and, thus, there are $N$ observations for each of the $K$ input variables. They are ordered in the table of random permutations of rank numbers which have $N$ rows and $K$ columns. Each row of the table is used on the $i$-th computer run. The simulation process is deterministic for a given set of generated rank numbers. One can evaluate the corresponding value $\mathrm{Y}_{\mathrm{n}}$ of the output for such a sample. From $N$ simulations, one can obtain a set of statistical data $\{Y\}=\left\{Y_{1}\right.$, $\left.\mathrm{Y}_{2}, \cdots, \mathrm{Y}_{\mathrm{n}}\right\}^{T}$. This set is statistically assessed so that the estimations of some statistical parameters, such as the mean value and the variance of the response, are obtained. Interested readers are referred to Oh and Yang, ${ }^{1}$ Novak et al. ${ }^{4}$ and Novak et al. ${ }^{8}$ for more details of this probabilistic method.

\section{Modification of Latin Hypercube sampling method}

\subsection{Sampling procedure}

LHS method can provide sufficient information about the uncertainties with a relatively small number of sampling. However, in generating an $N \times K$ design matrix, where $N$ is the sample size and $K$ is the number of variables, LHS may or may not give useful information about the uncertainty of the input variables because each value of an input variable is randomly selected from each interval of equal probability.

The improved statistical method proposed in this study is a modified LHS method in which the sampling method is more optimized than the conventional LHS method. This study presents a basic theoretical background to this improved variance reduction sampling scheme. This technique uses specially modified tables of random permutations of rank numbers which form the strategy of generating input samples for the simulation procedure. The methodto obtain these specially modified tables is described next.

The modified LHS method consists of two steps to obtain an $N \times K$ design matrix. The first step is dividing each input variable into $N$ intervals with equal probability of $1 / N$. The second step is the coupling of input variables with modified tables of random permutations of rank numbers. The general expression of the equation for the analytical model is shown as follows.

$Y=f(X)$

where, $Y=$ output variable

$f(\cdot)=$ deterministic analytical model

$X=$ the vector of input variables assumed to berandom ones $=\left[x_{1}, x_{2}, \cdots, x_{k}\right]^{\mathrm{T}}$

Every input variable $x_{k}(k=1,2, \cdots, K)$ is described by its known CDF with the appropriate statistical parameters. The sample $\{x\}_{n}$ of input variables, $n=1,2, \cdots, N(N$ being the number of sample equal to the number of simulations) is selected in the following way.

The range of known CDF $F_{X_{k}}(x)$ of each input variable $x_{k}$ is partitioned into $N$ disjunct intervals $S_{k n}$. Each interval is characterized by the probability $P_{k n}$ defined as

$$
P_{k n}=P\left(x_{k} \in S_{k n}\right)
$$

and

$$
\sum P_{k n}=1(k=1,2, \cdots, K)
$$

In the case of intervals of equal probability, it holds that $P_{k n}=1 / N$. Each interval is represented in the sample by the representative parameter, which is taken from the centroid of the interval. The representative parameter is obtained as

$$
F_{X_{k}}^{-1}\left(\frac{m_{n k}-0.5}{N}\right) \quad(k=1,2, \cdots, K)
$$

where, $F_{X_{k}}^{-1}(\cdot)=$ the inverse CDF

$$
\begin{aligned}
& m_{n k}=\text { the rank number of the interval used in } \\
& \text { the } n \text {-th simulation for input variable } x_{k}
\end{aligned}
$$

The representative parameter is used just once during the simulation procedure, and so there are $N$ observations for each of the $K$ input variables. $N$ observations for each of the input variable $x_{k}$ are associated with a sequence of integers (rank number of intervals), representing a random permutation of integers $1,2, \cdots$, $K$. They are ordered in the table of random permutations of rank numbers which have $N$ rows and $K$ columns. The rank numbers of intervals used in the $n$-th simulation are represented by the $n$-th row in the table. It means that this table forms the strategy for obtaining the input samples.

Tables used in LHS are commonly generated randomly. The possibility does exist that a certain statistical correlation among columns of the table is randomly introduced, which may have a significant influence on the results of simulation. Olsson et al. ${ }^{9}$ have introduced the correlation Latin Hypercube sampling plan which includes the correlation-reduction procedure. The correlation affects the bias and variance of the estimates obtained. Thus, it is required that rank permutations are mutually independent. To diminish the dependency of the input variables, some adjustment are made to $N \times K$ design matrix.

\subsection{Approach to non-parametric correlation}

There is a restrictive assumption of mutual independency between input variables. A measure of correlation between two parameters is the correlation coefficient.

Let the sampling model consist of a sample $\left(P_{1}, Q_{1}\right), \ldots,\left(P_{n}, Q_{n}\right)$.

$$
\left[P_{1}, P_{2}, \cdots, P_{n}\right]^{T} \text { and }\left[Q_{1}, Q_{2}, \cdots, Q_{n}\right]^{T}
$$

It is supposed that $P_{1} \leq \cdots \leq P_{n}$. Let $R_{1} \leq \cdots \leq R_{n}$ be the comresponding ranks of $Q_{1}, \cdots, Q_{n}$, then the corresponding rank array is as follows.

$$
[1,2, \cdots, n]^{T} \text { and }\left[R_{1}, R_{2}, \cdots, R_{n}\right]^{T}
$$

The Spearman rank-order correlation coefficient (the Spearman coefficient) can be defined as ${ }^{10}$

$$
r_{s}=\frac{\sum_{i=1}^{n}\left(i-\frac{n+1}{2}\right)\left(R_{i}-\frac{n+1}{2}\right)}{\sqrt{\sum_{i=1}^{n}\left(i-\frac{n+1}{2}\right)^{2} \sum_{i=1}^{n}\left(R_{i}-\frac{n+1}{2}\right)^{2}}}
$$

Since the ranks are a rearrangement of the integers from 1 to $n$, 
the denominator of eq. (7) can be reduced as follows.

$$
\sum_{i=1}^{n}(i-(n+1) / 2)^{2}=n\left(n^{2}-1\right) / 12
$$

and

$$
\sum_{i=1}^{n}(i-(n+1) / 2)=0
$$

Therefore, eq. (7) can be condensed as follows.

$$
r_{s}=1-\frac{6}{n\left(n^{2}-1\right)} \sum_{i=1}^{n}\left(i-R_{i}\right)^{2}
$$

The Spearman coefficient $r_{s}$ is always between -1 and +1 . When $r_{s}=1$, there is a perfect positive correlation between two variables; in other words, the increase of one variable increases the other. For $r_{s}=-1$, the reverse is true. The null hypothesis, that the correlation coefficient determined for a sample is a random deviation from the zero correlation in the population, is tested by means of the Student $t$ distribution with $n-2$ degrees of freedom. ${ }^{11}$ The $r_{s}$ to $t$ transformation equation is given as

$$
t=\left|r_{s}\right|\left(\frac{n-2}{1-r_{s}^{2}}\right)^{1 / 2}
$$

If $\mathrm{t} \geq t_{n-2, \alpha}$, the null hypothesis is rejected with significance level of $\alpha$ ( $\alpha$ is set usually at 0.05 ). That is, correlations exist between the variables that were two assumed be independent. The value of $r_{s}$ can also be judged on the basis of a $t$-distribution with $n-2$ degrees of freedom. The meaning of the value of $r_{s}$ is the same as that of the correlation coefficient explained previously. The assumption that two variables are independent can be tested by comparing $r_{s}$ with a critical value $r_{s}^{*}$. If $r \geq r_{s}^{*}$, the assumption is rejected with significance level of $\alpha$; in other words, there is dependency between the two variables.

In generating an $N \times K$ design matrix from input variables, the first columns of the $N \times K$ design matrix are random integers from 1 to $N$, i.e, the ranks of the first variable. In the next step, the second column is filled with random integers from 1 to $N$ to compute $r_{s 12}$, the rank correlation coefficient between $x_{1}$ and $x_{2}$. If $r_{s 12}$ is less than or equal to critical $r_{s}{ }^{*}$, fill the third column randomly with integers from 1 to $N$ and compute $r_{s 13}$ and $r_{s 23}$. If both $r_{s 13}$ and $r_{s 23}$ are less than or equal to critical $r_{s}{ }^{*}$, the next column is filled randomly again with integers from 1 to $N$. This procedure is repeated for all columns. After all columns are filled with integers from 1 to $N$, replace the integers of the $i$-th column by values of the $i$-th variable according to its magnitude.

\subsection{Matrix formulations for reducing undesired correlation}

Let $\mathbf{R}$ be an $N \times K$ matrix, of which columns represent $K$ permutations of integers $1,2, \ldots, N$. That is, matrix $\mathbf{R}$ is identical to the table of random permutations of rank numbers used in LHS schemes. Rank correlation among columns of this matrix is described by the rank correlation matrix $\mathbf{T}$, where element $T_{i j}, i, j=$ $1,2, \ldots, K$, are the Spearman coefficients among columns $i$ and $j$ of
$\mathbf{R}$. It is obvious that matrix $\mathbf{T}$ is symmetrical and is equal to the unit matrix I for the case of uncorrelated column. Consider the realizations of $\mathbf{R}$, for which matrix $T$ is positive definite, and let $\mathbf{S}$ be a lower triangular matrix such that

$$
\mathbf{S} \times \mathbf{T} \times \mathbf{S}^{\mathbf{T}}=\mathbf{I}
$$

where

$$
\mathbf{S}=\mathbf{Q}^{-1}
$$

Because matrix $\mathbf{T}$ is positive definite, the Cholesky factorization scheme can be used to find the lower triangular matrix $\mathbf{Q}$.

$$
\mathbf{T}=\mathbf{Q} \times \mathbf{Q}^{\mathrm{T}}
$$

The following transformation results in an $N \times K$ matrix $\mathbf{R}_{\mathbf{B} \text {. }}$

$$
\mathbf{R}_{\mathbf{B}}=\mathbf{R} \times \mathbf{S}^{\mathrm{T}}
$$

The statistical correlation among columns of this matrix is described by the rank correlation matrix $\mathbf{T}_{\mathbf{B}}$. Matrix $\mathbf{T}_{\mathbf{B}}$ should be close to $\mathbf{I}$. That is, the difference between appropriate elements in matrix $\mathbf{T}_{\mathbf{B}}$ and matrix $\mathbf{I}$ is smaller than the difference between the elements in matrix $\mathbf{T}$ and matrix $\mathbf{I}$. The values in each column of input matrix $\mathbf{R}$ can now be arranged so that they will have the same ordering as the corresponding column of matrix $\mathbf{R}_{\mathrm{B}}$. As the result, the rank correlation matrix $\mathbf{T}$ equals $\mathbf{T}_{\mathrm{B}}$, and the rank correlation among columns of $\mathbf{R}$ and also among columns of the table of random permutations of rank numbers is reduced.

The example of Table 1 shows how the rank correlation matrix T looks and how powerful the presented method can be. The table for $k=5$ input variables and $n=10$ simulation is randomly generated. Rank correlation among the columns of this table is described by the rank correlation matrix $\mathbf{T}$ as shown in Table 2 .

The extreme value of the Spearman coefficient is equal to -0.47 . The procedure described in this section is repeated twice, and columns of the table are rearranged. This improved the content of Table 1. The appropriate rank correlation matrix is shown in Table 2 . It is clearly seen that rank correlation among columns of the table is decreased. The extreme value of the Spearman coefficient is now equal to -0.07 .

Table 1 Random permutation of rank numbers for $k=5$ input variables and $n=10$ simulations.

\begin{tabular}{c|r|r|r|r|r|r|r|r|r|r}
\hline \multirow{2}{*}{$\begin{array}{c}\text { Simulation } \\
\text { (Run) }\end{array}$} & \multicolumn{4}{|c|}{ Non-modified rank } & \multicolumn{5}{c}{ Modified rank } \\
\cline { 2 - 11 } & \multicolumn{4}{|c|}{ Variables } & \multicolumn{5}{c}{ Variables } \\
\hline & 1 & 2 & 3 & 4 & 5 & 1 & 2 & 3 & 4 & 5 \\
\hline 1 & 1 & 3 & 4 & 1 & 5 & 1 & 3 & 4 & 2 & 4 \\
\hline 2 & 8 & 6 & 10 & 2 & 4 & 8 & 6 & 10 & 1 & 2 \\
\hline 3 & 5 & 5 & 9 & 3 & 7 & 5 & 5 & 9 & 6 & 5 \\
\hline 4 & 9 & 4 & 1 & 10 & 3 & 9 & 4 & 2 & 8 & 3 \\
\hline 5 & 6 & 10 & 7 & 8 & 1 & 6 & 10 & 5 & 9 & 1 \\
\hline 6 & 10 & 2 & 2 & 6 & 6 & 10 & 2 & 3 & 3 & 8 \\
\hline 7 & 2 & 1 & 5 & 9 & 10 & 2 & 1 & 7 & 10 & 6 \\
\hline 8 & 4 & 7 & 6 & 4 & 8 & 4 & 7 & 6 & 5 & 9 \\
\hline 9 & 7 & 8 & 8 & 7 & 9 & 7 & 8 & 8 & 7 & 10 \\
\hline 10 & 3 & 9 & 3 & 5 & 2 & 3 & 9 & 1 & 4 & 7 \\
\hline
\end{tabular}


Table 2 Rank correlation matrix of random permutationsof rank numbers from Table 1.

\begin{tabular}{c|c|c|c|c|c}
\hline \multirow{3}{*}{ Variables } & \multicolumn{5}{|c}{ Non-modified table } \\
\cline { 2 - 6 } & \multicolumn{5}{|c}{ Variables } \\
\cline { 2 - 6 } & 1 & 2 & \multicolumn{1}{c}{3} & \multicolumn{1}{c}{4} & \multicolumn{1}{c}{5} \\
\hline \hline 1 & 1.00 & 0.03 & -0.04 & 0.31 & -0.20 \\
\hline 2 & 0.03 & 1.00 & 0.37 & -0.03 & -0.47 \\
\hline 3 & -0.04 & 0.37 & 1.00 & -0.41 & 0.22 \\
\hline 4 & 0.31 & -0.03 & -0.41 & 1.00 & 0.01 \\
\hline 5 & -0.20 & -0.47 & 0.22 & 0.01 & 1.00 \\
\hline \multirow{3}{*}{ Variables } & \multicolumn{5}{|c|}{ Modified table } \\
\cline { 2 - 6 } & 1 & 2 & 3 & 4 & 5 \\
\hline \hline 1 & 1.00 & 0.03 & 0.02 & -0.07 & -0.07 \\
\hline \hline 2 & 0.03 & 1.00 & 0.01 & 0.05 & -0.04 \\
\hline 3 & 0.02 & 0.01 & 1.00 & -0.01 & -0.04 \\
\hline 4 & -0.07 & 0.05 & -0.01 & 1.00 & -0.03 \\
\hline 5 & -0.07 & -0.04 & -0.04 & -0.03 & 1.00 \\
\hline
\end{tabular}

\section{Uncertainty modeling of time-dependent properties of concrete}

Models for time-dependent properties or models for shrinkage and creep of concrete have been proposed by several researchers. Bazant and Panula ${ }^{12}$ suggest the use of the following equation for prediction of shrinkage strain. The details of the assumptions and underlying concepts of the model will not be discussed here in detail.

$$
\varepsilon_{s h}\left(t, t_{0}\right)=\Psi_{1} \varepsilon_{s h{ }^{\circ}} k_{h} S\left(t, t_{0}\right)
$$

where, $\varepsilon_{s h}\left(t, t_{0}\right)=$ shrinkage strain at any time $t ; \psi_{1}=$ model uncertainty factor for shrinkage model; $\varepsilon_{s h \infty}=$ ultimate shrinkage which is expressed by compressive strength of concrete, aggregate-cement ratio, sand-cement ratio, and water-cement ratio $; k_{h}=$ factor dependent on humidity; $S\left(t, t_{0}\right)=$ function dependent on size effect and aging effect ; $t=$ observation (current) time (in days); $t_{0}=$ the age of concrete starting to drying (in days).

The total creep function is the instantaneous strain plus both basic creep and drying creep strains at time $t$. Creep function is expressed by the following equation.

$$
J\left(t, t^{\prime}\right)=\Psi_{2}\left[\frac{1}{E_{0}}+C_{0} t, t^{\prime}\right]+\Psi_{3} C_{d}\left(t, t^{\prime}, t_{0}\right)
$$

where, $C_{0}\left(t, t^{\prime}\right)=$ basic creep coefficient at any time $t ; C_{d}\left(t, t^{\prime}\right.$, $\left.t_{0}\right)=$ drying creep coefficient ; $\Psi_{2}=$ model uncertainty factor for basic creep model ; $\Psi_{3}=$ model uncertainty factor for drying creep model.

The coefficients $\Psi_{1}, \Psi_{2}$, and $\Psi_{3}$ are model uncertainty factors. Information on model uncertainty can be found from the work of Bazant and Panula. ${ }^{12}$ Their study found that the coefficients of variation of shrinkage, basic creep and drying creep properties were $22 \%$ for shrinkage, $27 \%$ for basic creep, and $18 \%$ for drying creep, respectively. The reported coefficients of variation of the $\Psi$ factors are

$$
V_{\Psi_{1}^{*}}=0.22, \quad V_{\Psi_{2}^{*}}=0.27, \text { and } V_{\Psi_{3}^{*}}=0.18
$$

The coefficients, $\Psi_{1}^{*}, \Psi_{2}^{*}$ and $\Psi_{3}^{*}$, are prediction error terms that account for the uncertainty inherent in the theoretical model and the uncertainty of the micro-mechanism of creep and shrinkage that is not accounted for. The values in eq. (18) may be written as follows.

$$
\Psi_{1}^{*}=\Psi_{i} \Psi_{\alpha} \Psi_{\beta}(i=1,2,3)
$$

where, $\Psi_{i}=$ factor due to inadequacy of the prediction formula; $\Psi_{\alpha}=$ factor due to internal uncertainty; $\Psi_{\beta}=$ factor due to measurement errors and uncertainty in the laboratory (or field site) environment. The factors in eq. (19) are assumed independent, and the relation among the coefficients of variation is expressed as follows.

$$
\left(1+V_{\Psi_{1}}^{2}\right)=\left(1+V_{\Psi_{1}}^{2}\right)\left(1+V_{\Psi_{\alpha}}^{2}\right)\left(1+V_{\Psi_{\beta}}^{2}\right)(i=1,2,3)
$$

The results by Reinhardt et al. ${ }^{13}$ indicate that a value between 0.06 and 0.10 is reasonable for the estimation of $V_{\Psi_{\dot{\alpha}}}$ The coefficient of variation $V_{\Psi_{\beta}}$ was estimated as 0.05 by Madsen. ${ }^{14}$ The following corrected mean values and the coefficient of variation for model uncertainties $V_{i}$ are obtained from eqs. (18) and (20).

$$
\begin{array}{lll}
\text { Shrinkage } & : E\left[\Psi_{1}\right]=1 & \text { and } V_{\Psi_{1}}=0.20 \\
\text { Basic creep } & : E\left[\Psi_{2}\right]=1 & \text { and } V_{\Psi_{2}}=0.26 \\
\text { Drying creep } & : E\left[\Psi_{3}\right]=1 & \text { and } V_{\Psi_{3}}=0.16
\end{array}
$$

\section{Numerical analysis}

\subsection{Analytical model}

Creep and shrinkage of prestressed concrete structures are very complex phenomena in which various uncertainties exist with regard to material variations. Particular attention has been given to the uncertainty problem of creep and shrinkage. ${ }^{15-17} \mathrm{~A}$ probabilistic analysis to predict axial shortening of prestressed concrete box girders is carried out to investigate the effectiveness of the proposed method, and the statistical parameters of outputs are estimated. The span and cross-sectional geometry of girder for this numerical example are shown in Fig. 1. Axial shortening of prestressed concrete structure is time-dependent due to creep and shrinkage of concrete. Random input variables are chosen in terms of the uncertainty of time-dependent behavior of concrete

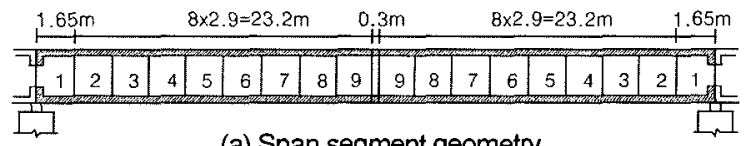

(a) Span segment geometry

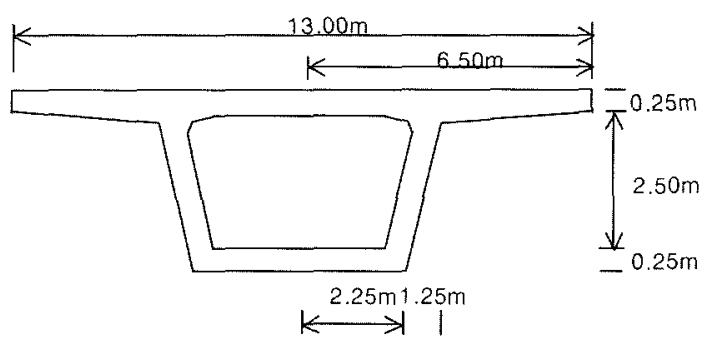

(b) Typical cross section

Fig. 1 Span and cross-sectional geometry 
structures. Shrinkage model uncertainty factor $\left(\Psi_{1}\right)$, uncertainty factor for basic creep $\left(\Psi_{2}\right)$, uncertainty factor for drying creep $\left(\Psi_{3}\right)$, relative humidity $(h)$, compressive strength of concrete $\left(f_{c}^{\prime}\right)$, aggregate-cement ratio $(a / c)$, sand-cement ratio $(s / c)$ and watercement ratio $(w / c)$ are assumed to be random variables. Each random variable is represented by its mean value and coefficient of variation. The statistical properties of input random variables are shown in Table 3 .

\subsection{Comparison of simulation results of modified LHS method and LHS method}

As it has been aforementioned in section 3.3, a decrease of the variance in the estimates of statistical parameters can be expected when modified LHS method is used. In this study, the estimates of mean value $\bar{Y}$, standard deviation $S_{Y}$, coefficient of variation (COV) $V_{y}$, skewness $a_{Y}$, minimum value in the sample $Y_{\mathrm{MIN}}$, and maximum value in the sample $Y_{M A X}$ obtained by LHS and modified LHS are compared.

Estimates are compared for 10,20 and 30 simulations. Sampling is repeated 10 sets for each number of simulations. The tables are randomly generated in every run for the case of LHS while the tables are rearranged and the statistical correlation of their columns is diminished for the case of modified LHS. Intervals have the same probability of $1 / \mathrm{N}$, and representative parameters are taken at the centroid of intervals.

After performing computer runs of ten sets, ten values of $\bar{Y}, S_{Y}$, $V_{y}, a_{Y}, Y_{\mathrm{MIN}}$ and $Y_{\mathrm{MAX}}$ are obtained. From theses particular samples, mean value $\bar{Y} z$, standard deviation $\mathrm{s}_{z}$, maximum value $\mathrm{MAX}_{Z}$ and minimum value $\mathrm{MIN}_{Z}$ are evaluated, where $Z=\bar{Y}, s_{Y}$, $v_{y}, a_{\mathrm{y}}, Y_{\mathrm{MAX}}$ and $Y_{\mathrm{MIN}}$. The value $\bar{Y} z$ is the estimate of the appropriate statistical parameter $Z$ of the basic sets, and the values $s_{z}, \mathrm{MAX}_{\mathrm{Z}}$ and $\mathrm{MIN}_{\mathrm{z}}$ characterize the variance of estimate and the amplitude of its possible values. When the statistics obtained by both sampling schemes are compared, the variance of estimates can also be compared.

The axial shortening of prestressed concrete box girder at 10,000 days after construction are presented in Fig. 2 through Fig. 7. The results obtained by LHS and modified LHS are plotted as a dashed line and a straight line, respectively, in the figures. Each figure consists of part (a) and part (b). In each figure, part (a) presents 3 plots-(1) mean value of an appropriate statistical parameter obtained from 10 sets, (2) minimum value of an appropriate statistical parameter obtained from 10 sets, and (3) maximum value of an appropriate statistical parameter obtained from 10 sets. The number of simulations and the value of appropriate parameter $Z$ are plotted on the horizontal and vertical axis, respectively. The statistics

Table 3 Statistical properties of input variables.

\begin{tabular}{cl|c|c|c}
\hline \multicolumn{2}{c|}{ Variables } & Mean & C.O.V. & Reference \\
\hline \hline$\psi_{1}$ & Uncertainty factor for shrinkage & 1.0 & 0.20 & 12 \\
\hline$\psi_{2}$ & Uncertainty factor for basic creep & 1.0 & 0.26 & 12 \\
\hline$\psi_{3}$ & Uncertainty factor for drying creep & 1.0 & 0.16 & 12 \\
\hline$h$ & Relative humidity (\%) & 61.6 & 0.27 & Measured \\
\hline$f_{c}^{\prime}$ & Concrete strength (MPa) & 49.2 & 0.07 & Measured \\
\hline$a / c$ & Aggregate-cement ratio & 3.44 & 0.1 & 14 \\
\hline$s / c$ & Sand-cement ratio & 1.40 & 0.1 & 14 \\
\hline$w / c$ & Water-cement ratio & 0.31 & 0.1 & 14 \\
\hline
\end{tabular}

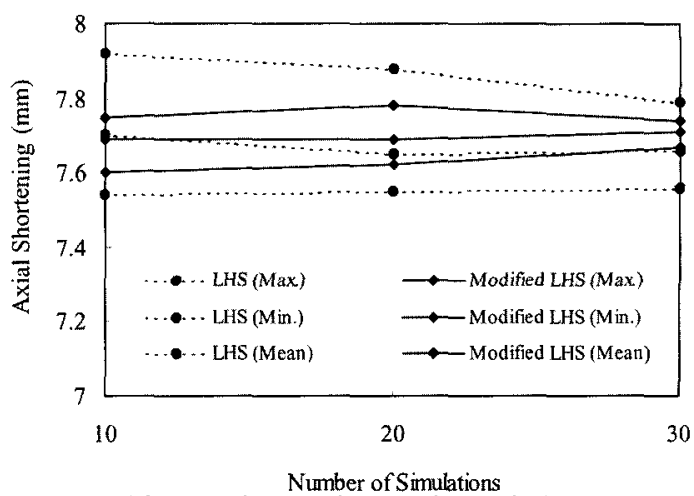

(a) Mean value, maximum value and minmum value

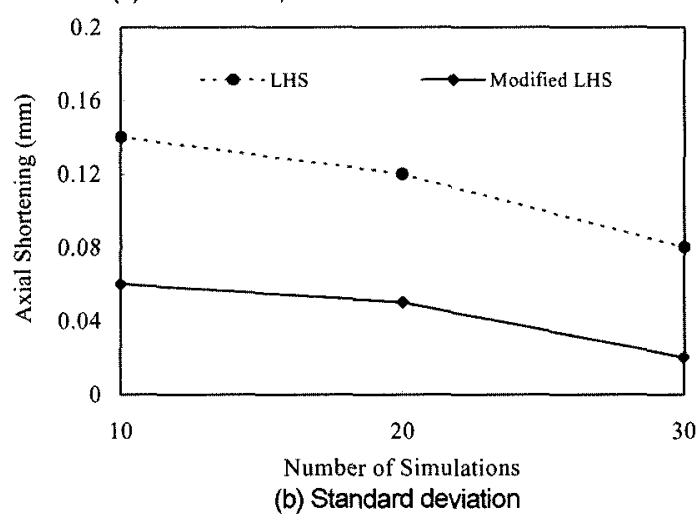

Fig. 2 Statistics of mean values.

for mean values is shown in Fig. 2. Mean values of modified LHS are almost the same as those of LHS. However, the difference between the maximum value and the minimum value determined by modified LHS is much smaller than the difference determined by LHS. Also, the standard deviation determined by modified LHS is much smaller than that determined by LHS

Statistics of standard deviations and statistics of coefficient of variation are shown in Figs. 3 and 4, respectively. Statistical characteristics of Figs. 3 and 4 are similar to those of Fig. 2. Mean values of modified LHS are almost the same as those of LHS. However, the difference between the maximum value and the minimum value determined by modified LHS is much smaller than the difference determined by LHS. The standard deviation determined by modified LHS is much smaller than that determined by LHS.

The statistics for skewness is shown in Fig. 5. Mean values of modified LHS are almost the same as those of LHS. Moreover, the difference between the maximum value and the minimum value determined by modified LHS is not much different from the difference determined by LHS. In addition, the standard deviation determined by modified LHS is is almost the same as the standard deviation determined by LHS.

Statistics of maximum values and minimum values are shown in Figs. 6 and 7, respectively. These two figures show that the maximum and minimum values as determined by the two respective methods used in this study did not differ significantly from each other. The standard deviation evaluated by modified LHS is smaller than that evaluated by LHS.

The results of the analysis show that the proposed method achieved a substantial reduction of the variance in the estimation of commonly used statistical parameters such as mean, standard 


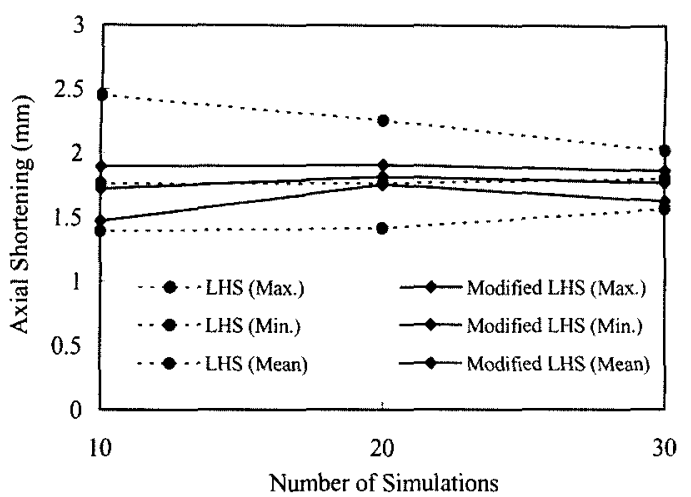

(a) Mean value, maximum value and minmum value

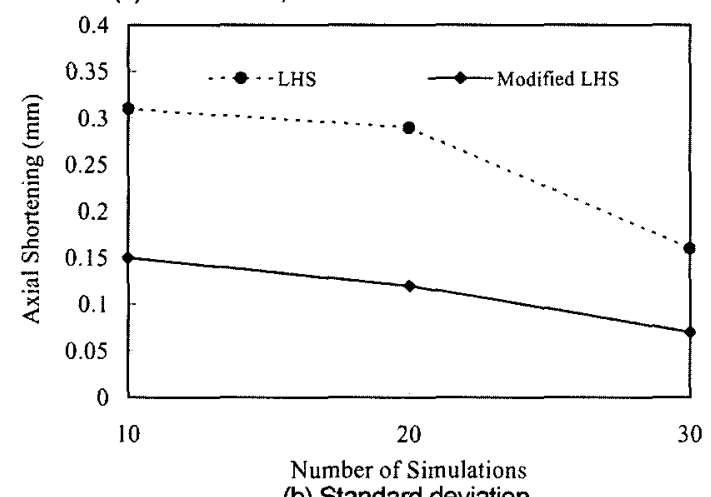

(b) Standard deviation

Fig. 3 Statistics of standard deviations.

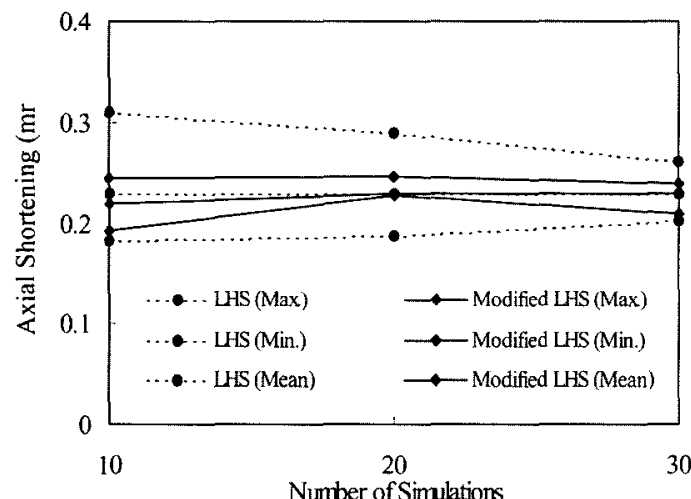

(a) Mean value, maximum value and minmum value

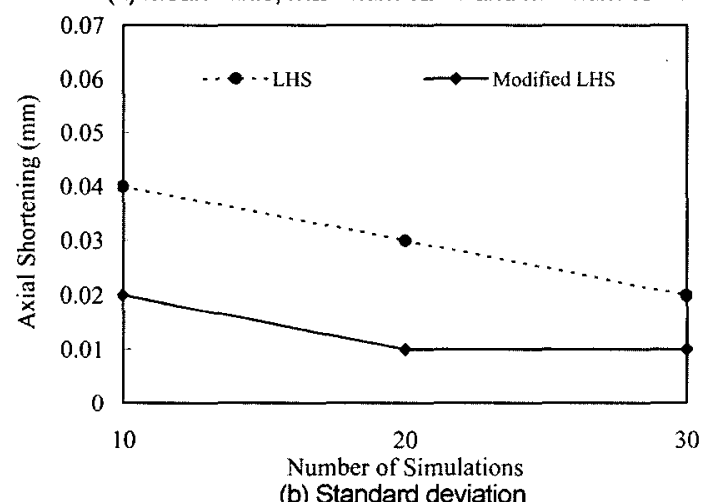

Fig. 4 Statistics of coefficient of variation.

deviation and coefficient of variation.

\section{Conclusions}

This paper presents an effective probabilistic analysis method

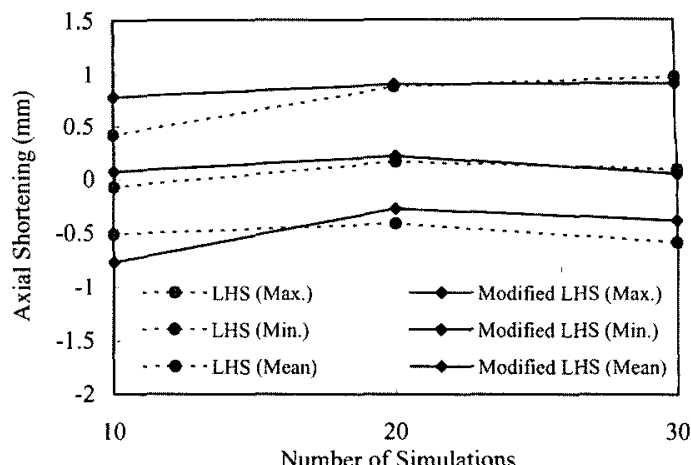

(a) Mean value, maximum value and minmum value

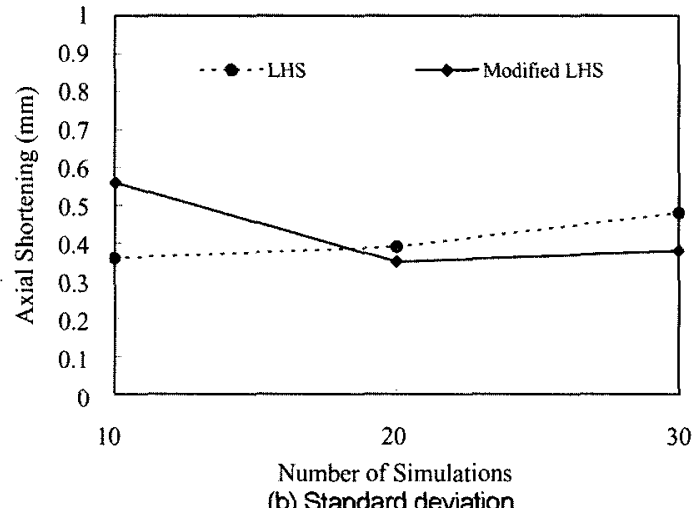

Fig. 5 Statistics of skewness.

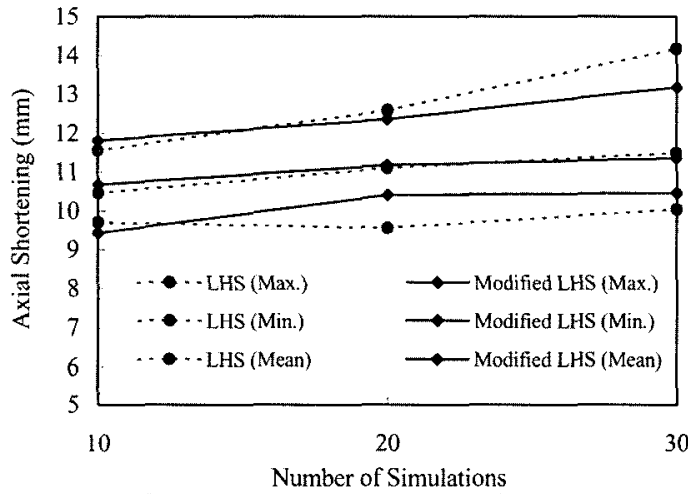

(a) Mean value, maximum value and minmum value

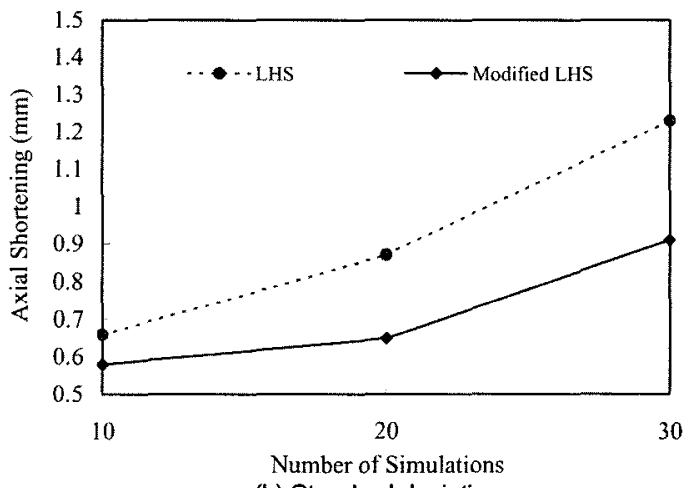

(b) Standard deviation

Fig. 6 Statistics of maximum values.

for the uncertainty analysis of concrete structures. It is a modification of Latin Hypercube sampling method. The proposed method uses specially modified tables in which bias of rank number is decreased. Spearman rank correlation coefficient is applied to decrease the bias of rank correlation. As an application 


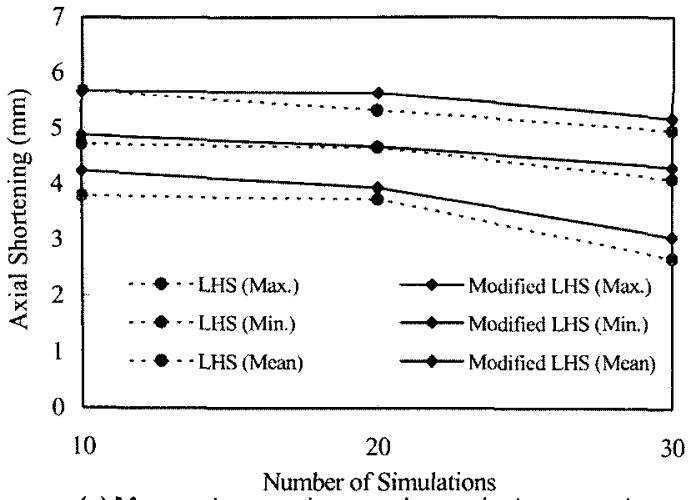

(a) Mean value, maximum value and minmum value

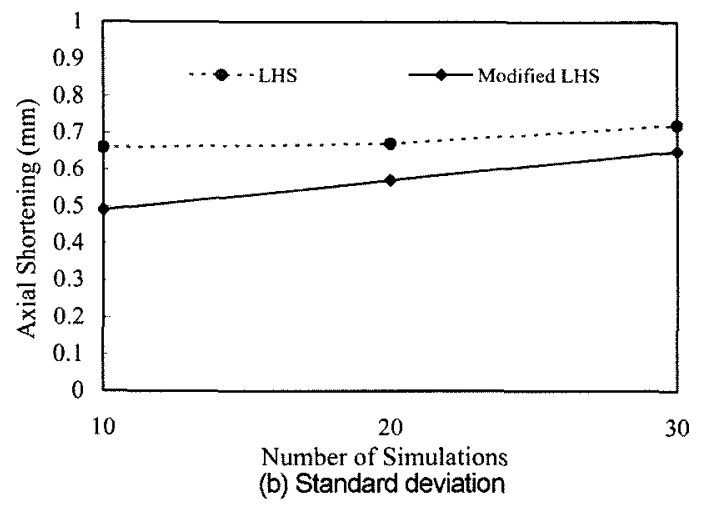

Fig. 7 Statistics of minimum values.

example, a numerical analysis of pre-stressed concrete box girder is carried out to analyze its axial deformation. The application study revealed that the difference between the maximum and minimum values, standard deviation, and coefficient of variation determined by the proposed method was much smaller than those determined by conventional LHS method. Thus, it follows that the proposed method results in a significant reduction of the variance in the estimates of common statistical parameters. Finally, the proposed probabilistic analysis method is expected to be effective in the uncertainty analysis of such structures as concrete bridges. Future study with regard to the application of the proposed method will attest for its effectiveness in the field application even more.

\section{References}

1. Oh, B. H. and Yang, I. H., "Sensitivity Analysis of TimeDependent Behavior in PSC Box Girder Bridges," Journal of Structural Engineering, ASCE, Vol.126, No.2, 2000, pp.171 179.

2. Fossum, A. F. and Munson, D. E., "Probabilistic Creep Analysis of Underground Structures in Salt," Journal of Engineering Mechanics, ASCE, Vol.122, No.3, 1996, pp.209 217.

3. Madsen, H. O., Krenk, S., and Lind, N. C., Methods of
Structural Safety, Prentice Hall, Englewood Cliffs, New Jersey, 1986.

4. Novak, D., Teply, B., and Shiraishi, N., "Sensitivity Analysis of Structures : a review," Proceedings of CIVIL COMP 93, Edinburgh, Scotland, 1993, pp.201 207.

5. Iman, R. L. and Conover, W. J., "Small Sample Sensitivity Analysis Techniques for Computer Models with an Application to Risk Assessment," Communications in Statistics, A9, 1980, pp. $1749 \sim 1842$.

6. Iman, R. L., Helton, J. C., and Campbell, J. E., "An Approach to Sensitivity Analysis of Computer Models, Part I Introduction, Input Variable Selection and Preliminary Variable Assessment," Journal of Quality Technology, Vol.13, No.3, July, 1981, pp. $174 \sim 183$.

7. Iman, R. L., Helton, J. C., and Campbell, J. E., "An Approach to Sensitivity Analysis of Computer Models, PartRanking of Input Variables, Response Surface Validation, Distribution Effect and Technique Synopsis," Journal of Quality Technology, Vol.13, No.4, Oct., 1981, pp.232 240.

8. Novak, D., Teply, B., and Kersner, Z., "The Role of Latin Hypercube Sampling Method in Reliability Engineering," Proceedings of ICOSSAR-97, Kyoto, Japan, 1997, pp.403 409.

9. Olsson, A., Sandberg, G., and Dahlblom, O., "On Latin Hypercube Sampling for Structural Reliability Analysis," Structural Safety, 25, 2003, pp.47 68.

10. Hettmansper, T. P., Statistical Inference Based on Ranks, John Wiley \& Sons, New York, 1984.

11. Scheaffer, R. L. and McClave, J. T., Probability and Statistics for Engineers, 2nd Ed., Duxbury Press, Boston, 1986.

12. Bazant, Z. P. and Panula, L., "Creep and Shrinkage Characterization for Analyzing Prestressed Concrete Structures," PCI Journal, Vol.25, No.3, 1980, pp.86 122.

13. Reinhardt, H. W., Pat, M. G., and Wittmann, F. H., "Variability of Creep and Shrinkage of Concrete," Proceedings of symposium on fundamental on creep and shrinkage of concrete, M.Nijhoff, The Hague, 1982, pp.75 94.

14. Madsen, H. O. and Bazant, Z. P., "Uncertainty Analysis of Creep and Shrinkage Effects in Concrete Structures," $A C I$ Journal, Vol.80, March-April, 1983, pp.116 127.

15. Diamantidis, D., Madsen, H. O., and Rackwitz, R., "On the Variability of the Creep Coefficient of Structural Concrete," Materials and Structures, Vol.17, No.100, 1983, pp.321 328.

16. Li, C. Q. and Melchers, R. E., "Reliability Analysis of Creep and Shrinkage Effects," Journal of Structural Engineering, ASCE, Vol.118, No.5, 1992, pp.1532 1548.

17. Khor, E. H., Rosowsky, D. V., and Stewart, M. G., "Probabilistic Analysis of Time-dependent Deflections of RC Flexural Members," Computers \& Structures, Vol.79, Issue. 16, 2001, pp.1461 1472. 